\title{
Using Low-level Currents Measured during Lightning Events to Estimate Upward Leader Properties
}

\author{
Marley Becerra \\ School of Electrical Engineering and Computer Science \\ KTH Royal Institute of Technology \\ Stockholm, Sweden \\ marley@kth.se
}

Marcelo M. F. Saba

National Institute for Space Research - INPE

São José dos Campos, SP, Brazil

marcelo.saba@inpe.br

\begin{abstract}
Low-level currents measured prior to return strokes can potentially provide information about the properties of upward leaders during lightning flashes. However, these currents need to be properly analysed and interpreted in order to be useful for evaluating upward connecting leaders. In this paper, low-level currents measured before return strokes in two lightning events to two structures in Brazil are analysed and interpreted as case studies. The discharge current estimated from one of these events is used as input to a detailed thermohydrodynamic model with an extensive kinetic scheme for $\mathrm{N}_{2} / \mathrm{O}_{2}$ mixtures. The model allows the evaluation of the physical and chemical properties of upward connecting leaders. Estimates of the temperature, mass density, electric field and radius of the channel are presented for an upward connecting leader propagating in a lightning event. In addition, estimates of the axial density of electrons, ions and neutral particles (including NO and $\mathrm{NO}_{2}$ ) are also reported.
\end{abstract}

Keywords: leaders, thermohydrodynamic model, lightning

\section{INTRODUCTION}

Measurements of lightning currents in instrumented structures are traditionally aimed to record the return stroke peak current. For this reason, they usually have a minimum resolution of more than a few hundred amperes, which is not sufficient to resolve the low-level currents produced by other events during lightning strikes. This is the case of the currents produced during the inception and propagation of upward leaders under thunderstorms, which are in the order of less than several tens of amperes.

Fortunately, there is a growing number of instrumented structures and rocket-triggered-lightning experiments measuring the low-level currents prior to return strokes (e.g.

\author{
Lipeng Liu \\ School of Electrical Engineering and Computer Science \\ KTH Royal Institute of Technology \\ Stockholm, Sweden \\ lipeng@kth.se
}

Silverio Visacro

Lightning Research Center

UFMG: Federal University of Minas Gerais

Belo Horizonte, Brazil

Silverio_visacro@hotmail.com

[1]- [4] ). Even though some of these measurements have been used to evaluate the striking distance [1], they could also provide important additional data to analyse the physical properties of upward connecting leaders. However, these measured low-level currents are composed not only by the current produced by the electrical discharges produced under thunderstorms. Measurements have shown that current measured from vertical conductors also include an induction current component produced by electromagnetic coupling to the downward discharge [3]. On the other hand, the current of upward leaders prior to the onset of their stable propagation (also known as stable leader inception) has been predicted to be in the order of few amperes [5]. Thus, they are comparable with the minimum resolution of reported low-level current measurements [1] [2] indicating a low signal-to-noise ratio. For these reason, the usage of these measurements is not trivial and requires careful engineering and physical analysis.

In this paper, the first attempt to use low-level currents measured in instrumented structures for estimating the properties of upward connecting leaders is reported. Low-level currents measured prior to return stroke lightning events to a 14-story apartment building in Sao Paulo and to an instrumented tower in Morro do Cachimbo Station in Brazil are analysed and interpreted. The current estimated from one of these events is used as input to a detailed thermohydrodynamic model for estimating the typical physical and chemical properties of an upward connecting leader in a lightning event

\section{CAlCUlating UpWARd LEADER PROPERTIES FROM CURRENT MEASUREMENTS}

The physical process of initiation and propagation for leader discharges has been recently calculated with a detailed one-dimensional thermohydrodynamic model with an extensive kinetic scheme for N2/O2 mixtures [6] [7]. For this model, the current of a single thermalized channel is used as

M.B would like to acknowledge the financial support of the financial support of the Swedish strategic research program StandUp for Energy. 
input to evaluate the thermal and chemical properties of streamer stems and leaders as a function of time. The evaluation is based on the solution of the equations for conservation of mass, momentum and energy for overall translational and vibrational states in humid air, together with equations describing the kinetics of 45 species participating in 192 chemical reactions. The electric field inside the leader channel is calculated using ohm's law as a function of the measured current and the temperature-dependent gas conductivity of the channel along the radial direction.

Even though positive leaders have already analysed under laboratory conditions [6] [7], it is relevant to evaluate the physical properties of upward leaders in nature. However, one significant challenge is to use such a thermohydrodynamic model within a sufficiently long simulation time and spatial computational domain as to reproduce the conditions under thunderstorms. Due to the significantly larger magnitude, variations and duration of the currents flowing through leaders under thurderstorms (as it will be shown in next Section), numerical instabilities can occur at any instant of the simulation.

\section{LOW-LEVEL CURRENT ANALYSIS}

\section{A. Event $20160224 P 2$}

The first case study considered corresponds to the low-level current produced by an unsuccessful upward positive leader launched from an instrumented building P2 on the $24^{\text {th }}$ of February 2016, at 18:48:16 in Sao Paulo, Brazil. The building was $46 \mathrm{~m}$ tall and it was protected by a $6 \mathrm{~m}$ tall lightning rod. The rod was instrumented with a Pearson current monitor with low and high frequency cutoff of $5 \mathrm{~Hz}$ and $2 \mathrm{MHz}$ respectively [5]. In addition, electric field was measured $4 \mathrm{~m}$ away from the rod with a fast antenna. The antenna decay constant was $0.5 \mathrm{~ms}$ and its high frequency cut-off is $1.5 \mathrm{MHz}$. Both the current and the electric fields were recorded continuously using a $200 \mathrm{~ns}$ time resolution. High speed photography footage for that event was also available.

Fig. 1.a shows the raw measured low-level current for the analysed event. As it can be seen, the recorded current has a natural noise lower than $\pm 5 \mathrm{~A}$. The signal has also an average offset value of about 1.7 A several milliseconds before the return stroke. Digital processing of the signal is performed using a low-bandpass filter with cutoff of $2 \mathrm{MHz}$ and the offset has been subtracted. The filtered signal shows a slowly increasing background component with superimposed peaks.

Further evaluation of the electric field measurements shows that the recorded $\mathrm{dE} / \mathrm{dt}$ signal (shown in Fig. 1.b) has peaks at the same time and with similar waveform as the current pulses measured through the lightning rod. This result indicates that the lightning rod may be also acting as a dE/dt antenna. Interestingly, a similar effect has been observed for a $7 \mathrm{~m}$ tall conductor in [3]. Analysis of the high speed photography footage also shows a visible change in the area in front of the lightning rod at the moment when the current peaks are detected. For these reasons, it can be concluded that the current pulses (and some oscillations around the background) shown in Fig. 1.a can be partially caused by electromagnetic coupling with the descending downward stepped leader, rather than produced by the upward leader. However, it is difficult to estimate the actual contribution of this antenna effect from the current measurements.

Even though the thermalization (and unstable formation) of a leader segment will be evident by a sudden increase in the current from zero level [4], such change cannot be clearly observed from the raw or filtered current signals in this event. Due to the limited resolution the current measurement, the sudden current change (of few amperes or less) could not be observed probably due to low signal to noise ratio. In order to circumvent this limitation, the high speed photography footage could be used to define the instant when the first leader channel was detected. This time coincided with the instant when the first current pulse was observed (at $-0.56 \mathrm{~ms}$ in Fig. 1.a). Since this first detected leader was already 3 meters long, it is unlikely that the unstable leader inception occurred at this time. Thus, it is not possible to conclusively define the leader inception time. For this reason, the recorded current cannot be used as input of the thermodynamic model for further analysis of the upward leader properties.
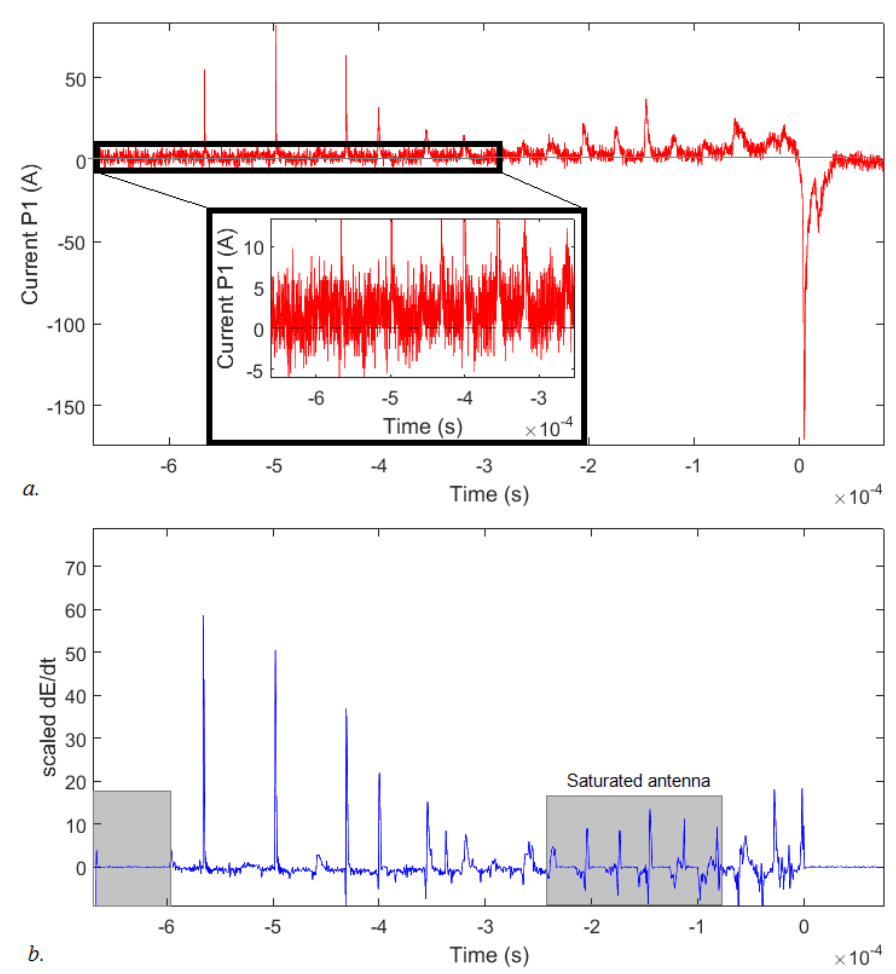

Figure 1. Recorded data for the event 20160224P1: a) low level current, $b$ ) scaled $\mathrm{dE} / \mathrm{dt}$

\section{B. Event MCS20140225}

The second case study corresponds to the low-level current produced by a successful upward connecting positive leader launched from an instrumented mast on the $25^{\text {th }}$ of February 2015 [1]. The tower is $60 \mathrm{~m}$ tall and it stands on top of a mountain $1430 \mathrm{~m}$ over sea level. The mast is equipped with a 
current monitor with cutoff frequencies of $3 \mathrm{~Hz}$ and $1.5 \mathrm{MHz}$ located at its base. The current is recorded with a $16.7 \mathrm{~ns}$ resolution with a $15 \mathrm{~ms}$ pretrigger. The noise level in the raw measurement of $\pm 5 \mathrm{~A}$ was reduced to $\pm 2 \mathrm{~A}$ by processing the signal as described in [1] .

Fig. 2 shows the raw current recorded during this event. As it can be seen, there is a sudden current jump at $-1.42 \mathrm{~ms}$, followed a well-defined slowly increasing background component. This instant can be clearly defined as the unstable leader inception in that event. The measured current also shows current pulses superimposed to the background component. Unfortunately, it is not possible to correlate these current pulses with the electric field change. Even though electric field measurements are available for the mast, they were sampled with a coarser resolution of 500 ns for that event. However, it has been shown that the peak of these pulses is correlated to the amount of charge deposited along the downward leader [8]. Thus, as in the event discussed in III.A, it is likely that these current peaks are partially caused by the electromagnetic coupling between the mast/leader system and the descending stepped leader. Since it is difficult to quantify the contribution of this antenna effect on the upward leader alone, it is assumed as a first approximation that these current peaks are not flowing directly through the leader channel. Thus, the upward leader current is defined by the lower-frequency current component as shown in Fig. 2.

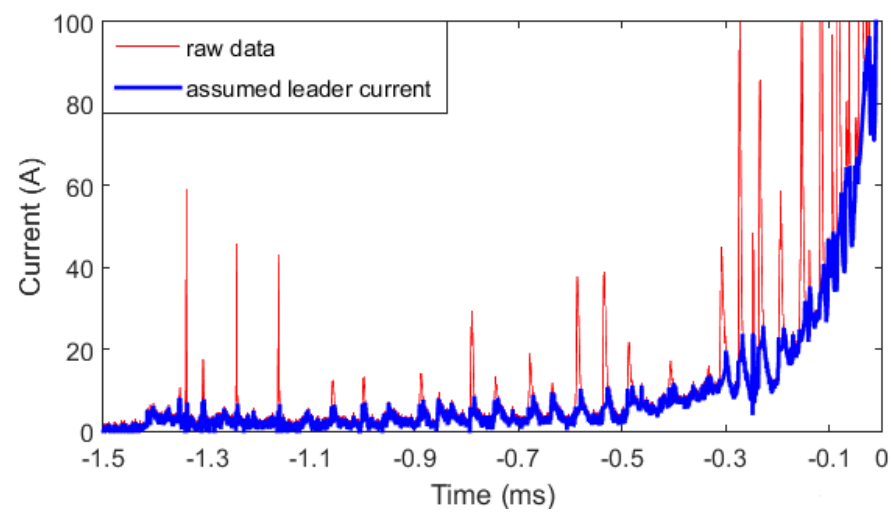

Figure 2. Recorded raw current data for the event MCS20140225 and the estimated upward leader current. The zero time is the instant of the first return stroke.

It is important to highlight that streamer discharges must have occurred before the unstable inception of the leader. In the laboratory, the current pulses associated to streamers have risetimes in the order of 5 to $15 \mathrm{~ns}$ and half-decay times between 50 and $150 \mathrm{~ns}$ [6]. Typical peak values of those current pulses have been estimated with the Self-consistent Leader Inception Model (SLIM) to be in the order of few to few tens of amperes [4] [9]. However, no such current pulses are visible from the measurements before the leader onset. This unfortunate fact can be caused by the limited frequency bandwidth of the current measurements. Observe that an upper cutoff frequency of only $1.5 \mathrm{MHz}$ could smooth out and reduced the streamer current peaks to magnitudes lower than the noise level.

\section{UPWARD CONNECTING LEADER PROPERTIES FOR THE EVENT MCS20140225}

The simulation of the chemical properties and radial heating dynamics of the upward connecting leader in the event MCS20140225 are simulated using as input the filtered current waveform shown in Figure 2. Since the streamer current pulses occurring prior to the streamer inception cannot be detected, the simulation is started from the leader inception time at $-1.42 \mathrm{~ms}$. A typical initial channel radius of $0.2 \mathrm{~mm}$ is assumed as in [6] [7].

\section{A. Leader physical properties}

Figure 3 shows the simulated radial gas heating dynamics of the leader channel at several time instants. As can be seen, the dynamics of the connecting leader soon after its unstable inception are similar to those obtained for leaders in the laboratory [6] [7]. Soon after the leader current starts, the density around the channel in this event reaches values lower than 0.1 times the atmospheric density $\rho_{a m b}$. Since the pressure after the first microsecond recovers to atmospheric conditions, temperature increases accordingly according to the gas law. Even though the leader channel expands rapidly during the first tens of microseconds after inception, its radial velocity decreases to less than $10 \mathrm{~m} / \mathrm{s}$ as the current flows through the leader.
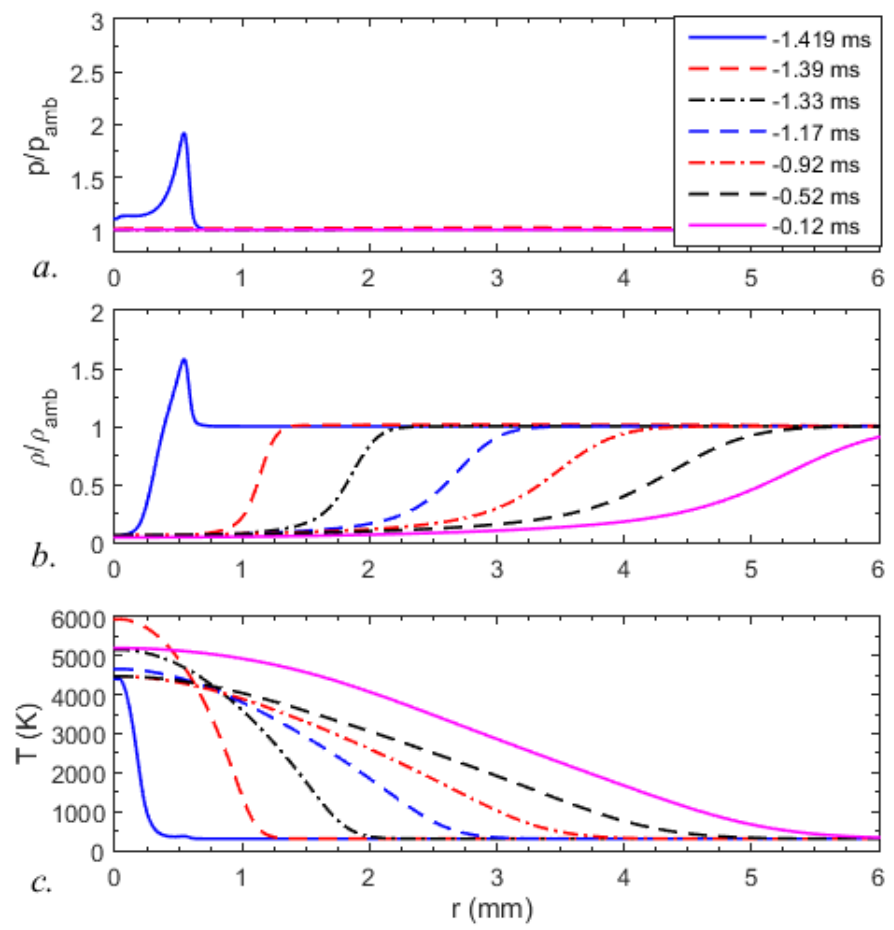

Figure 3. Radial gas dynamics of (a) relative pressure, (b) relative air density and (c) gas temperature. 
The simulated time variation of the density and particle temperatures of the leader channel at the axis of symmetry is shown in Figure 4 as a function of time. Interestingly, the gas heating in this event is estimated to occur under a rather constant axial density (around $0.06 \rho_{a m b}$ ) and gas temperature $T$ of about $4500 \mathrm{~K}$ during the leader propagation period until $-0.3 \mathrm{~ms}$ before the return stroke. Despite of current variations ranging between about 2 to $20 \mathrm{~A}$, the changes in the injected energy during this period did not lead to significant changes in the axial gas properties of the leader. Since the leader channel has not reached yet thermodynamic equilibrium, any variation in current does not lead directly to a gas temperature change. Instead, the change in current causes directly variations of the electronic $T_{e}$ and vibrational $T v$ temperatures.
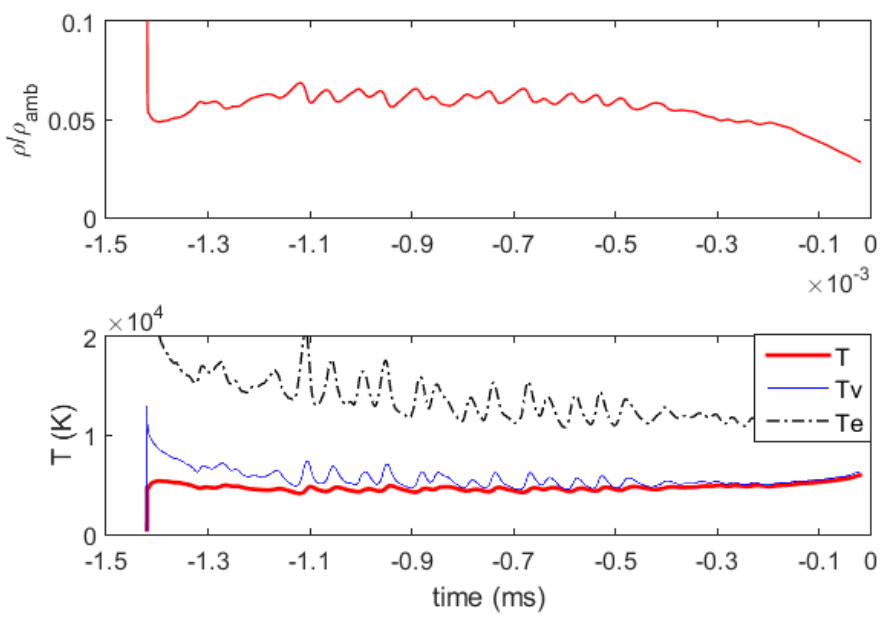

Figure 4. Estimated axial relative air density, electron temperature $T_{e}$, vibrational temperature $T_{v}$ and gas temperature $T$ of the leader channel as a function of time.

The thermodynamic detailed model also allows estimations of the thermal radius and electric field along the leader in the event. Figure 5 shows the comparison of these parameters calculated with the detailed thermohydrodynamic model and those calculated based on Gallimberti's theory [10] and the Bazelyan and Raizer semi-empirical equations [11]. Observe that the results of Gallimbert's theory are strongly dependent on the chosen initial leader electric field $E_{0}$. Although the differences in the channel radius here calculated and obtained with Gallimberti's theory are lower than $30 \%$, the disagreement in the leader electric field is significantly larger. In this event, the equation of Bazelyan and Raizer shows a fair agreement with the calculated leader field during the period when the leader is in non-thermal equilibrium. After this period, the electric fields predicted according to [11] are grossly underestimated.

\section{B. Leader chemical properties}

Figure 6 shows the density of the main species in the leader channel axis. Even though complex reactions occur during the first microsecond after the unstable leader inception (not visible in Figure 6), the charged particles in the leader become mainly dominated by electrons and NO positive ions during the subsequent leader development, with densities ranging between $10^{14}$ and $10^{16}$ particles $/ \mathrm{cm}^{3}$. During this period, the composition of the leader axis is dominated by NO, with density of about $4 \cdot 10^{17}$ particles $/ \mathrm{cm}^{3}$. As thermal equilibrium starts at about $-0.3 \mathrm{~ms}$ and the axial temperature increases, the composition of $\mathrm{NO}$ in the leader axis decreases by increased dissociation into $\mathrm{O}$ and $\mathrm{N}$. However, $\mathrm{NO}$ continues to be produced in off-centered areas of the leader channel, where the gas temperature is lower. Since the concentration of $\mathrm{NO}_{2}$ and $\mathrm{O}_{3}$ is several orders of magnitude lower than for $\mathrm{NO}$, they are not included in Figure 6.
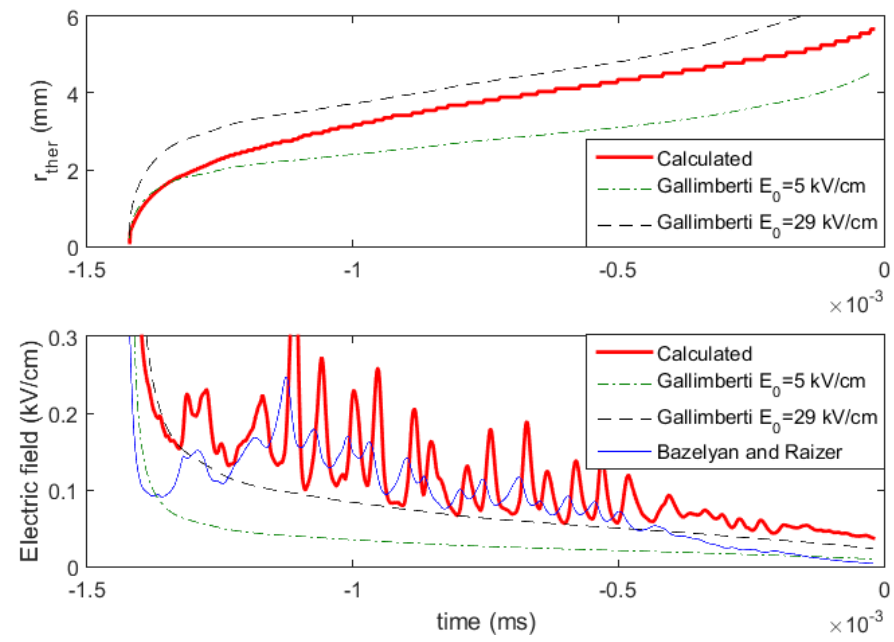

Figure 5. Estimated thermal radius and electric field of the leader channel. Calculations performed using Gallimberti's theory and Bazelyan and Raizer equations are also included for sake of comparison.
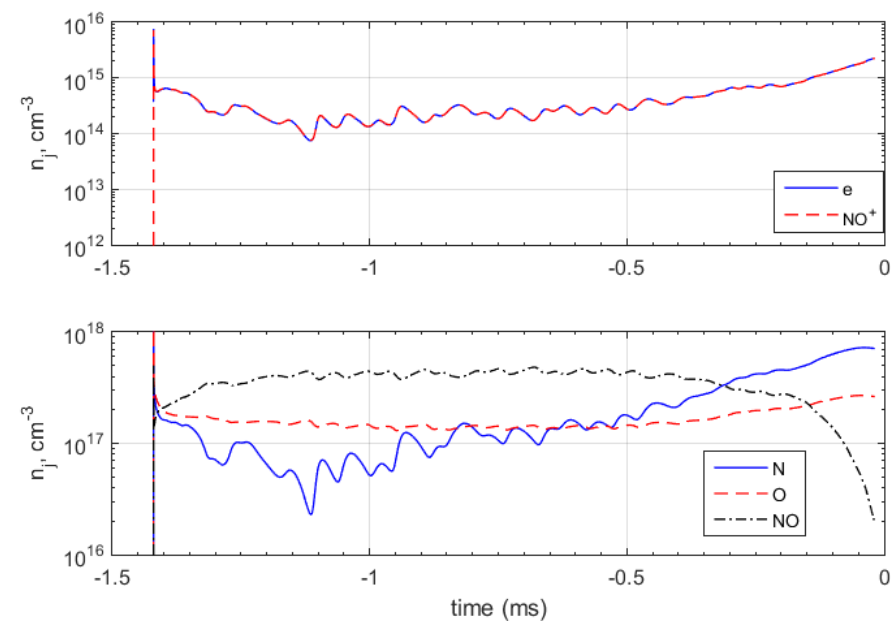

Figure 6. Estimated axial density of charged particles as a function of time. 


\section{CONCLUSIONS}

Low-level currents measured prior to two lightning events are analysed and interpreted as input to a detailed thermohydrodynamic model to calculate the physical and chemical properties of upward connecting leaders. Using one of those events as a case study, the radial gas dynamics of the leader channel are reported. Comparison of the thermal radius and electric field of the leader channel estimated with the model with estimates based on the theory of Gallimberti and the semi-empirical equation of Bazelyan and Raizer has been performed. Furthermore, the chemical properties of the leader axis are presented, showing that NO dominates at the axis of symmetry.

\section{ACKNOWLEDGMENT}

M.B would like to acknowledge the financial support of the financial support of the Swedish strategic research program StandUp for Energy.

\section{REFERENCES}

[1] S. Visacro, M. Guimaraes and M. H. M. Vale, "Striking Distance Determined From High-Speed Videos and Measured Currents in Negative Cloud-to-Ground Lightning," Journal of Geophysical Research: Atmospheres, vol. 122, no. 24, p. 13356-13369, 2017.

[2] J. D. Hill, M. A. Uman, D. M. Jordan, T. Ngin, W. R. Gamerota, J. Pilkey and J. Caicedo, "The attachment process of rocket-triggered lightning dart-stepped leaders," J. Geophys. Res. Atmos., vol. 121, p. 853-871, 2016.

[3] J. Schoene, M. A. Uman, V. A. Rakov, J. Jerauld, B. D. Hanley, K. J. Rambo, J. Howard and B. DeCarlo, "Experimental Study of Lightning-Induced Currents in a Buried Loop Conductor and a Grounded Vertical Conductor," IEEE IEEE TRANSACTIONS ON ELECTROMAGNETIC COMPATIBILITY, vol. 50, no. 1, pp. 110-117, 2008.

[4] M. Becerra, "Corona discharges and their effect on lightning attachment," Atmospheric Research, vol. 149, p. 316-323, 2014.

[5] M. M. F. Saba, A. R. Paiva, C. Schumann, M. A. S. Ferro, K. P. Naccarato, J. C. O. Silva, F. V. C. Siqueira and D. M. Custódio, "Lightning attachment process to common buildings," Geophysical Research Letters, vol. 44, no. 9, p. 4368-4375, 2017.

[6] L. Liu and M. Becerra, "On the critical charge required for positive leader inception in long air gaps," Journal of Physics D: Applied Physics, vol. 51, p. 035202, 2018.

[7] L. Liu and M. Becerra, "Gas heating dynamics during leader inception in long air gaps at atmospheric pressure," Journal of Physics D: Applied Physics, vol. 50, no. 34, p. 345202, 2017.

[8] S. Visacro, M. H. Murta, M. Guimaraes and A. Teixeira, "Early phase of lightning currents measured in a short tower associated with direct and nearby lightning strikes," Journal of Geophysical Research, vol. 115, no. D16104, pp. 1-13, 2010.

[9] M. Becerra and V. Cooray, "A self-consistent upward leader propagation model," J. Phys. D: Appl. Phys., vol. 39, pp. 3708$3715,2006$.

[10] I. Gallimberti, "The mechanism of long spark formation," $J$. Phys. Coll, vol. 40, p. 193-250, 1972.

[11] E. Bazelyan and Y. Raizer, Lightning Physics and Lightning Protection, Bristol: Institute of Physics, 2000. 